\title{
Outcomes and Prognosis of Neurological Decompression and Stabilization for Spinal Metastasis: Is Assessment with the Spinal Instability Neoplastic Score Useful for Predicting Surgical Results?
}

\author{
Kenji Masuda ${ }^{1}$, Ko Ebata ${ }^{1}$, Yoshimasa Yasuhara ${ }^{1}$, Akira Enomoto ${ }^{1}$, Tomoyuki Saito ${ }^{2}$ \\ ${ }^{1}$ Department of Orthopaedic Surgery, Yokosuka Kyosai Hospital, Yokosuka, Japan \\ ${ }^{2}$ Department of Orthopaedic Surgery, Yokohama City University School of Medicine, Yokohama, Japan
}

\begin{abstract}
Study Design: Retrospective study.
Purpose: To evaluate the efficacy of the Spinal Instability Neoplastic Score (SINS) in predicting surgical outcomes and survival. Patients were categorized into two groups according to the SINS, and their surgical outcomes and survival following decompression and stabilization were assessed.

Overview of Literature: Palliative surgery in patients with a life expectancy $\geq 3$ months may effectively improve their overall condition in the long term. Currently, the effectiveness of the SINS for predicting surgical results and survival remains controversial.

Methods: This study included 44 patients who underwent decompression and stabilization for spinal metastases at Yokosuka Kyosai Hospital between 2008 and 2017. The patients were divided into two groups: stable (SINS $\leq 12$ ) and unstable (SINS $\geq 13$ ). Changes in the Frankel score and Eastern Cooperative Oncology Group Performance Status (ECOG-PS) were compared between the two groups, and patient survival was evaluated according to the SINS, Tokuhashi score, and Katagiri score.

Results: The stable group (SINS range, 7-12) included 24 patients while the unstable group (SINS range, 13-16) included 20 patients. The Frankel score significantly improved from 2.8 to 3.6 in the stable group $(p<0.001)$ and from 2.7 to 3.9 in the unstable group $(p<0.001)$. The ECOG-PS significantly improved from 3.2 to 2.1 in the stable group $(p<0.001)$ and from 3.0 to 1.8 in the unstable group $(p<0.001)$. There was a statistically significant difference in median survival between the two groups.

Conclusions: All patients treated with palliative surgery showed favorable outcomes, as indicated by improved the Frankel score and ECOG-PS following surgery. However, median survival was significantly better in the stable group. The results of this study indicate that the SINS is appropriate for surgical decision making and may be used to predict survival.
\end{abstract}

Keywords: Spinal instability neoplastic score; Spinal neoplasms; Palliative surgery; Spinal fusion; Prognosis

Received Nov 5, 2017; Revised Feb 17, 2018; Accepted Mar 19, 2018

Corresponding author: Kenji Masuda

Department of Orthopaedic Surgery, Yokosuka Kyosai Hospital, 1-16 Yonegahama-dori, Yokosuka 238-0011, Japan

Tel: +81-46-822-2710, Fax: +81-46-825-2103, E-mail: mashmash4952@msn.com 


\section{Introduction}

The skeleton is the most common site of metastasis in advanced cancer, with the spine being the most frequent location [1]. Approximately $30 \%$ of all patients with advanced cancer develop spinal metastasis [2]. The surgical management of spinal metastasis is a widely considered palliative intervention but prognosis remains poor, with survival times commonly ranging from 3 months to $<1$ year after surgery [2-4]. When a patient's spine is determined to be unstable, surgical stabilization prior to radiotherapy or systemic therapy is appropriate. The purpose of surgery is pain control and the preservation/restoration of function. This is achieved by the aggressive decompression of the spinal cord at the site of major compression, followed by stabilization. Studies have demonstrated that surgical management improves the neurological function and quality of life $[5,6]$. Similarly, evidence in the literature suggests reasonable long-term survival following surgery for spinal metastasis [5-7].

The Spinal Instability Neoplastic Score (SINS), which has demonstrated near-perfect inter- and intra-observer reliability in determining three clinically relevant categories of stability, can guide the need for surgical assessment (Table 1) [8-12]. Although the SINS is a reliable tool for spinal surgeons and radiologists while rating tumorrelated spinal instability, the reliability of the SINS in determining survival prognosis remains controversial [1315]. Therefore, the present study assessed the outcomes and survival of patients after surgical decompression and stabilization according to the SINS.

\section{Materials and Methods}

\section{Patient population and study criteria}

This study was approved by the Ethical Research Board of Yokosuka Kyosai Hospital (approval no., 17-14). A retrospective review of all patients surgically treated for the management of spinal metastasis at our institution between 2008 and 2017 was conducted. Patients with spinal metastasis who underwent surgery met the following criteria: medical stability with rapidly progressive neurological deterioration and/or radiographic instability. Patients were excluded from surgical treatment if they were not sufficiently stable for surgery or if their projected life expectancy was $<3$ months, as assessed by the oncologist.
Table 1. Spinal Instability Neoplastic Score

\begin{tabular}{ll}
\hline Spinal Instability Neoplastic Score component & Score \\
\hline Location & \\
\hline Junctional (occiput-C2, C7-T2, T11-L1, L5-S1) & 3 \\
\hline Mobile spine (C3-C6, L2-L4) & 2 \\
\hline Semi-rigid (T3-T10) & 1 \\
\hline Rigid (S2-S5) & 0 \\
\hline Pain) & \\
\hline Yes & 3 \\
\hline Occasional pain but not mechanical & 1 \\
\hline Pain-free lesion & 0 \\
\hline Bone lesion & \\
\hline Lytic & 2 \\
\hline Mixed (lytic/blastic) & 1 \\
\hline Blastic & 0 \\
\hline Radiographic spinal alignment & \\
\hline Subluxation/translation present & 4 \\
\hline De novo deformity & 2 \\
\hline Normal alignment & 0 \\
\hline Vertebral body collapse & 3 \\
\hline$>50 \%$ collapse & 2 \\
\hline$<50 \%$ collapse & 1 \\
\hline No collapse with $>50 \%$ body involved & 0 \\
\hline None of the above & 3 \\
\hline Posterolateral involvement of spinal elements & \\
\hline Bilateral & \\
\hline Unilateral & \\
\hline None of the above & \\
\hline
\end{tabular}

alPain improvement with recumbency and/or pain with movement/ loading of spine. b) Facet, pedicle, or costovertebral joint fracture or replacement with tumor.

During the study period, 44 patients ( 30 males and $14 \mathrm{fe}$ males) underwent surgery for spinal metastasis and were included in this study. All patients provided informed consent for their participation in the study. The mean age at the time of surgery was $66.5 \pm 9.2$ years (range, $47-81$ years), and the mean follow-up duration was $28.3 \pm 32.7$ months (range, 1.0-128.0 months) after surgery. Medical records were reviewed for cancer type, prior chemotherapy, total burden of skeletal and spinal metastases, visceral metastasis, surgical procedures, radiography, and blood analysis results.

Functional status was assessed preoperatively and postoperatively using the Eastern Cooperative Oncology 
Table 2. The Eastern Cooperative Oncology Group Performance Status grading system

\begin{tabular}{ll}
$\begin{array}{l}\text { Grade } \\
0\end{array}$ & $\begin{array}{l}\text { Cully active, able to carry out all pre-disease performance without restriction } \\
\text { Restricted in physically strenuous activity but ambulatory and able to carry out work of a light or sedentary nature, e.g., light house } \\
\text { work, office work }\end{array}$ \\
1 & Ambulatory and capable of self-care but unable to carry out any work activities up and about $>50 \%$ of waking hours \\
\hline 3 & Capable of only limited self-care, confined to bed or chair more than $50 \%$ of waking hours \\
\hline 4 & Completely disabled, cannot carry out any self-care, totally confined to bed or chair more than $50 \%$ of waking hours \\
\hline 5 & Dead
\end{tabular}

Table 3. Comparison of demographic and clinical characteristics of patients in the stable and unstable groups treated with decompression and stabilization for spinal metastases

\begin{tabular}{|c|c|c|c|}
\hline Characteristic & Stable group & Unstable group & $p$-value \\
\hline Spinal Instability Neoplastic Score & $24(7-12)$ & $20(13-16)$ & \\
\hline \multicolumn{4}{|l|}{ Sex } \\
\hline Male & $14(58)$ & $16(80)$ & \\
\hline Female & $10(42)$ & $4(20)$ & \\
\hline Age at surgery (yr) & $67 \pm 9.1(50-79)$ & $66 \pm 9.5(47-81)$ & NS \\
\hline Follow-up period (mo) & $34 \pm 36.0$ & $21 \pm 27.4$ & NS \\
\hline Preop Tokuhashi score & $8.2 \pm 2.8$ & $6.9 \pm 3.2$ & NS \\
\hline Preop Katagiri score & $4.3 \pm 2.0$ & $5.2 \pm 1.9$ & NS \\
\hline Preop Frankel score & $2.8 \pm 0.7$ & $2.7 \pm 0.9$ & NS \\
\hline Preop Eastern Cooperative Oncology Group Performance Status & $3.2 \pm 0.8$ & $3.0 \pm 1.1$ & NS \\
\hline
\end{tabular}

Values are presented as number (range), number (\%), mean \pm SD (range), or mean \pm SD.

SD, standard deviation; NS, not significant; Preop, preoperative.

Group Performance Status (ECOG-PS) grading system (Table 2). Neurological function was assessed according to the Frankel score. Spinal instability was assessed using the SINS. Patients were stratified into three categories of stability on the basis of the total SINS: stable (0-6 points); potentially unstable (7-12 points); and unstable (13-18 points). For the purpose of this study, the patients were divided into two groups. Those with SINS $\leq 12$ were assigned to the stable group, while those with SINS $\geq 13$ were assigned to the unstable group. Demographic characteristics of the two groups are presented in Table 3. Improvements in the Frankel score and ECOG-PS were analyzed and compared between the two groups. The Frankel scores were converted from A to E into a scale from 1 to 5 , respectively. Patient survival was then evaluated according to the SINS, as well as the Tokuhashi and Katagiri scores (Tables 4, 5) [16,17].

The Tokuhashi score grades six radiographs in combi- nation with clinical components for a total score ranging from 0 to 15 [16]. Patients with life expectancies $<6$ months (score $0-8$ ) are treated with conservative management or palliative surgery; those with a life expectancy between 6 and 12 months (score 9-11) are candidates for palliative surgery; those with a life expectancy $>12$ months (score 12-15) are treated with excision surgery.

The Katagiri score sums six clinical components for a total score ranging from 0 to 10 [17]. The prognosis of survival of patient with a score ranging from 7 to 10 is significantly poorer than that of patients with a score ranging from 0 to 6 .

Postoperative survival as a function of time was expressed using Kaplan-Meier estimates, with death as the failure event. Survival curves were compared using the log-rank test. 
Table 4. The Tokuhashi scoring system for the preoperative evaluation of metastatic spine tumor prognosis

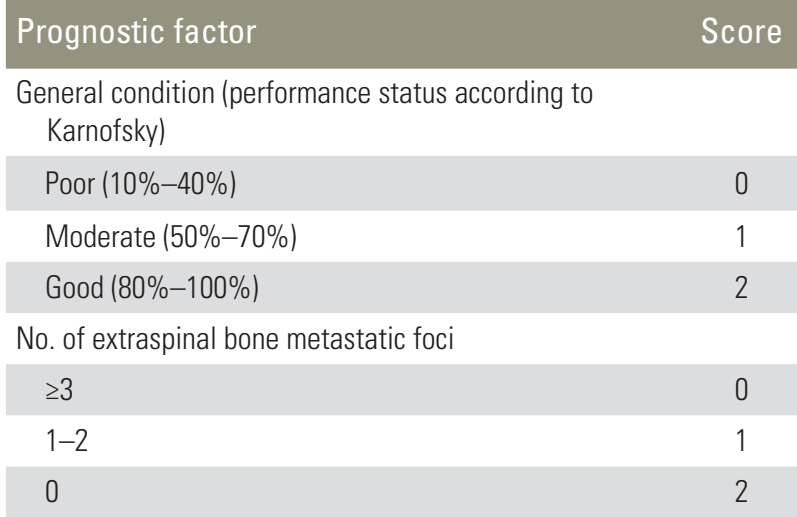

No. of metastases in the vertebral bodies

\begin{tabular}{|lc|}
\hline$\geq 3$ & 0 \\
\hline 2 & 1 \\
\hline 1 & 2 \\
\hline Metastases to the major internal organs & 0 \\
\hline Irremovable & 1 \\
\hline Removable & 2 \\
\hline No metastases & \\
\hline Primary site of cancer & 0 \\
\hline Lung, stomach & 1 \\
\hline Kidney, liver, uterus, unidentified, other & 2 \\
\hline Thyroid, prostate, breast, rectum & 0 \\
\hline Spinal cord palsy & 1 \\
\hline Complete & 2 \\
\hline Incomplete & \\
\hline None & \\
\hline
\end{tabular}

Interpretation of the Tokuhashi score: 9-12, radical surgery; 6-8, clinical judgment; $0-5$, palliative management.

\section{Statistical analysis}

Survival statistics and Kaplan-Meier curves were calculated using the IBM SPSS ver. 24.0 (IBM Corp., Armonk, NY, USA). Undefined median survival indicates that more than half of the patient cohort remained alive throughout the follow-up period; thus, median survival was never reached. The threshold for statistical significance was set at $p<0.05$. Paired and unpaired $t$-tests were performed using Microsoft Excel 2013 (Microsoft Corp., Redmond, WA, USA).

\section{Results}

Of the 44 patients, 43 underwent the posterior decompression and stabilization of one to three spinal levels, while one patient underwent anterior decompression and stabilization.

In total, 24 patients with the SINS between 7 and 12 were categorized into the stable group. Of the 24 patients in the stable group who underwent surgery for spinal metastasis, five $(20.8 \%)$ presented with lung cancer, four $(16.7 \%)$ with prostate cancer, three $(12.5 \%)$ with renal cell carcinoma, two $(8.3 \%)$ with breast cancer or thyroid cancer, and eight (33.4\%) with cancer types categorized as "other." Metastases were observed in the cervical spine in five patients, in the thoracic spine in 14 patients, and in the lumbar spine in five patients. Visceral metastasis was observed in 10 patients $(41.7 \%)$.

In total, 20 patients with the SINS between 13 and 16 were categorized into the unstable group. Of the 20 patients who underwent surgery for spinal metastasis, eight (40\%) presented with lung cancer, four (20\%) with prostate cancer, two (10\%) with breast cancer, and six (30\%) with cancer types categorized as "other." Metastases were observed in the cervical spine in three patients, in the thoracic spine in 13 patients, and in the lumbar spine in four patients. Visceral metastasis was observed in 11 patients (55\%).

The Tokuhashi and Katagiri scores were 8.2 and 4.3 in the stable group and 6.9 and 5.2 in the unstable group, respectively. The Tokuhashi and Katagiri scores were not significantly different between the groups (Table 3 ). The differences in the preoperative Frankel score and ECOG-PS were not statistically significant between the groups. The Frankel score significantly improved from 2.8 to 3.6 in the stable group $(p<0.001)$ and from 2.7 to 3.9 in the unstable group $(p<0.001)$ (Fig. 1). The ECOGPS significantly improved from 3.2 to 2.1 in the stable group $(p<0.001)$ and from 3.0 to 1.8 in the unstable group $(p<0.001)$ (Fig. 2). The period from the manifestation of symptoms to surgery was 28 and 17 days in the stable and unstable groups, respectively (not statistically significant).

The 6-month overall survival rates after surgery in the stable and unstable groups were $80 \%$ and $37 \%$, respectively. The median survival of patients in the stable group was undefined. The median survival of patients in the unstable group was 14 months. There was a statistically significant difference in median survival between the groups $(p=0.01$, log-rank test) (Fig. 3). 
Table 5. The Katagiri scoring system for patients with skeletal metastasis

\section{Prognostic factor}

Primary site

Slow growth: hormone-dependent breast and prostate cancer, thyroid cancer, multiple myeloma, and malignant lymphoma

Moderate growth: lung cancer treated with molecularly targeted drugs, hormone-independent breast and prostate cancer, renal cell carcinoma, endometrial and ovarian cancer, sarcoma, and others

Rapid growth: lung cancer without molecularly targeted drugs, colorectal cancer, gastric cancer, pancreatic cancer, head and neck cancer, esophageal cancer, other urological cancer, melanoma, hepatocellular carcinoma, gall bladder cancer, cervical cancer, and cancers of unknown origin

Visceral metastasis

Nodular visceral or cerebral metastasis 1

Disseminated metastasis ${ }^{\text {a) }}$

Laboratory data

Abnormal $^{\text {bl }}$

1

Critical $^{\mathrm{c})}$

2

Eastern Cooperative Oncology Group Performance Status: 3 or 4

Previous chemotherapy

Multiple skeletal metastases

Total

alPleural, peritoneal, or leptomeningeal dissemination. ${ }^{\mathrm{b}} \mathrm{C}$-reactive protein $\geq 0.4 \mathrm{mg} / \mathrm{dL}$, lactate dehydrogenase $\geq 250 \mathrm{IU} / \mathrm{L}$, or serum albumin $<3.7 \mathrm{~g} /$

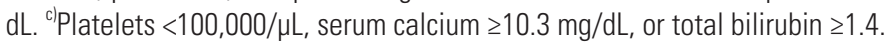

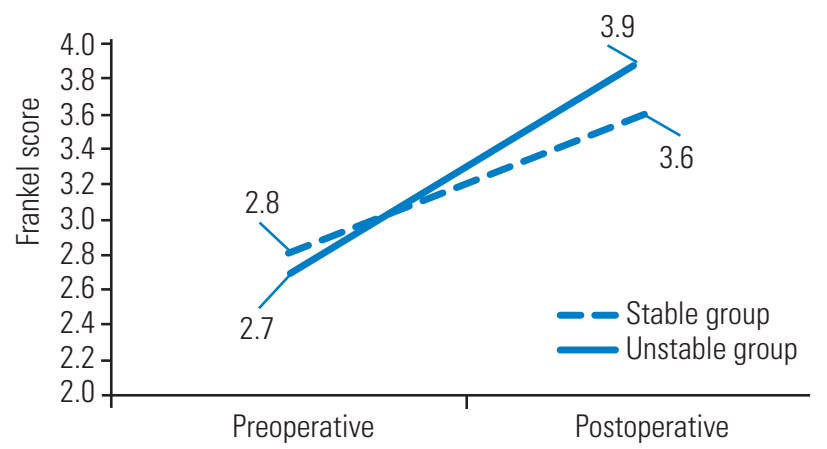

Fig. 1. The improvements of Frankel score. The Frankel score improved significantly in both groups.

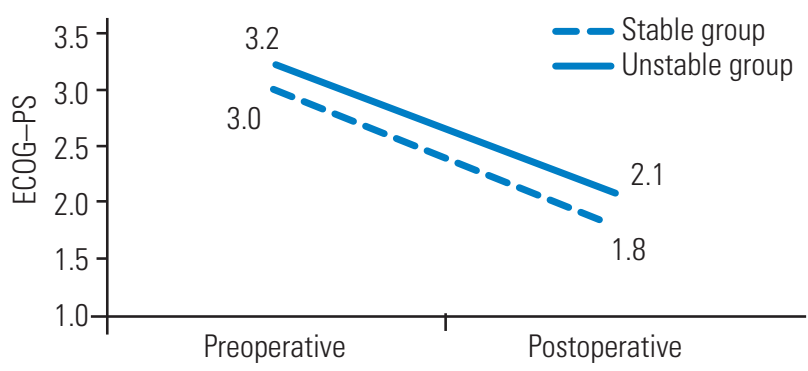

Fig. 2. The improvements of ECOG-PS. The ECOG-PS score improved significantly in both groups. ECOG-PS, Eastern Cooperative Oncology Group Performance Status.

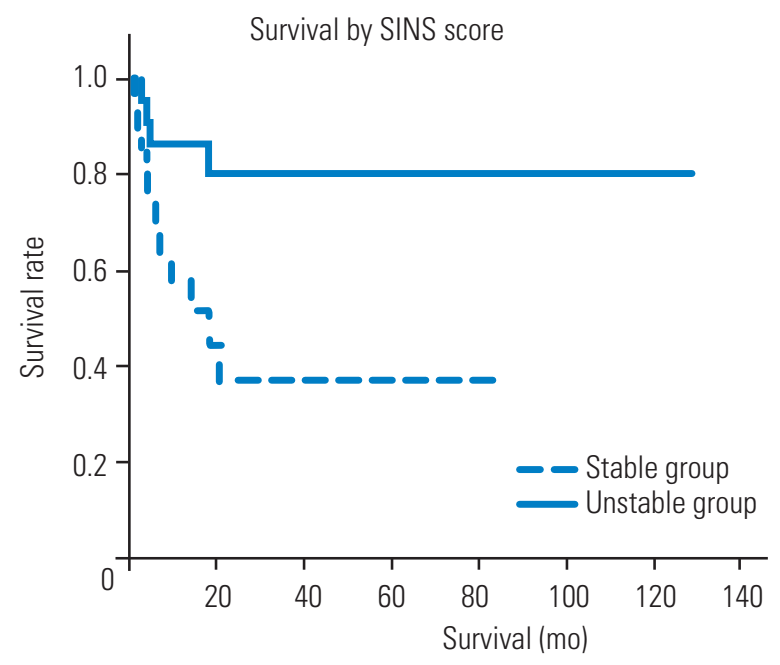

Fig. 3. Kaplan-Meier survival curve for SINS. There was a statistically significant difference in median survival between the two groups. SINS, Spinal Instability Neoplastic Score.

The 6-month overall survival rates after surgery in patients with a Katagiri score $\geq 7$ and $\leq 6$ were $0 \%$ and $66 \%$, respectively. The median survival of patients with a Katagiri score $\geq 7$ was 3 months. The median survival of patients with a Katagiri score $\leq 6$ was undefined. There was a statistically significant difference in the median survival of these patients ( $p=0.04$, log-rank test) (Fig. 4). 


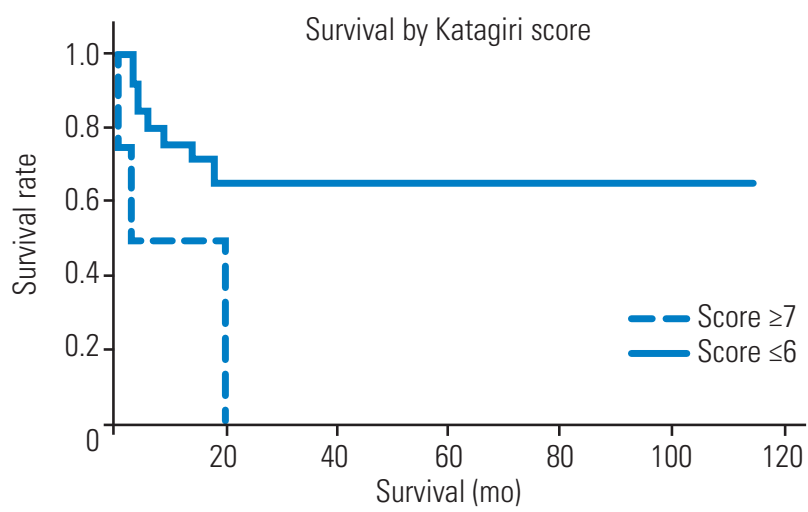

Fig. 4. Kaplan-Meier survival curve for Katagiri score. There was a statistically significant difference in median survival between the two groups.

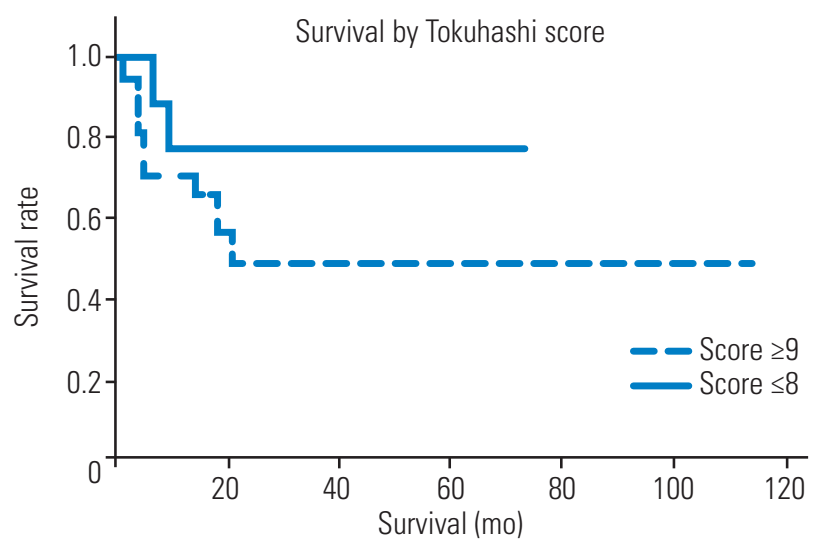

Fig. 5. Kaplan-Meier survival curve for Tokuhashi score. There was no statistically significant difference in median survival between the two groups.

The 6-month overall survival rates after surgery in patients with a Tokuhashi score $\geq 9$ and $\leq 8$ were $78 \%$ and $49 \%$, respectively. The median survival of patients with a Tokuhashi score $\geq 9$ was undefined. The median survival of patients with a Tokuhashi score $\leq 8$ was 11 months. There was no statistically significant difference in the median survival of these patients ( $p=0.23$, log-rank test) (Fig. 5).

\section{Discussion}

The survival time of patients with bone metastasis has substantially improved over the last two decades due to advances in chemotherapy and radiotherapy. These developments have increased the frequency at which such cases are referred for spinal surgery [18].

Scores related to prognosis and life expectancy have been modified to adjust for prolonged survival times $[16,17]$. The appropriate surgical management of spinal metastasis provides recovery from neurological dysfunction and severe pain related to tumor compression. The aim of palliative surgery, achieved through the aggressive decompression of the neural elements and stabilization of the spinal column, is the improvement of neurological function and activities of daily living.

In this study, patients were divided into the stable and unstable groups according to their SINS, and the outcomes of spinal cord stabilization in both groups were assessed. Hussain et al. [19] have reported that surgical stabilization significantly improved clinical outcomes of patients with indeterminate and unstable SINS. Similarly, Cavalcante et al. [20] have reported that surgical stabilization led to significant improvement in pain and neurological status in patients with higher SINS.

Patients in both the groups demonstrated improvements in the Frankel score and ECOG-PS following surgery. Thus, the purpose of surgical decompression and stabilization was accomplished in both the groups. In previous studies, the SINS was used as a binary indicator for surgical referral: stable (0-6 points) or current/possible instability (7-18 points). Surgical consultation is recommended for patients with a score $\geq 7$. Palliative surgery for all patients in this study whose SINS ranged from 7 to 17 was performed for improving patient comfort and function, and the patients met the criteria for surgical recommendation [8].

Zadnik et al. [15] have reported that although the SINS predicts spinal instability and identifies suitable patients for surgical management, it does not predict survival after surgery. Studies have investigated the prognostic value of the SINS for survival after surgery but did not demonstrate prognostic value for postoperative survival $[13,15]$.

However, in the present study, there was a statistically significant difference in the median survival between the stable and unstable groups. Moreover, there was a statistically significant difference in the median survival between the two groups according to the Katagiri score. Tancioni et al. [21] have reported that the ECOG-PS, the period from the manifestation of symptoms to surgery, and the presence of visceral metastases affected functional outcomes and survival in patients treated for vertebral metastases. According to primary tumor histology distribution, the incidence of lung cancer was elevated in the unstable group compared with that in the stable group. The preoperative ECOG-PS, period from the manifestation of symptoms to surgery, pathological level, and the 
rate of visceral metastases were not significantly different between the groups. However, since the SINS (which evaluates local disease status) and the background of both the groups were similar beyond the primary tumor distribution, spinal instability may have influenced the prognosis.

A limitation of this study is the selection bias during enrollment. This study included only patients who underwent surgical treatment. Accordingly, patients with relatively high SINS (range, 7-16) were recruited. Both higher and lower SINS may have precluded patients from surgical treatment (deemed insufficiently stable for surgery or having a projected life expectancy of $<3$ months). If patients who received conservative treatment and those who showed a low SINS (range, 0-6) were included the study, we may have obtained different results. Thus, this selection bias of patients limits the generalizability of this study beyond the studied patient population.

Additionally, the higher incidence of lung cancer in the unstable group compared with that in the stable group is another limitation of this study. The primary tumor type is an important factor included in the prognostic scoring system, which affects the postoperative survival time. Therefore, the higher incidence of lung cancer in the unstable group may have led to a worse prognosis in the unstable group compared with that in the stable group.

\section{Conclusions}

Patients with spinal metastasis, who were divided into the stable and unstable groups according to the SINS and treated with surgical neurological decompression and stabilization, showed improvements in the Frankel score and ECOG-PS. However, the survival of patients in the unstable group was shorter than that in the stable group, indicating that the SINS may have a prognostic value.

\section{Conflict of Interest}

No potential conflict of interest relevant to this article was reported.

\section{Author Contributions}

Kenji Masuda wrote and prepared the manuscript, and all of the authors participated in the study design. All authors have read, reviewed, and approved the article.

\section{References}

1. Coleman RE. Clinical features of metastatic bone disease and risk of skeletal morbidity. Clin Cancer Res 2006;12(20 Pt 2):6243s-6249s.

2. Wibmer C, Leithner A, Hofmann G, et al. Survival analysis of 254 patients after manifestation of spinal metastases: evaluation of seven preoperative scoring systems. Spine (Phila Pa 1976) 2011;36:1977-86.

3. Walker MP, Yaszemski MJ, Kim CW, Talac R, Currier BL. Metastatic disease of the spine: evaluation and treatment. Clin Orthop Relat Res 2003;(415 Suppl):S165-75.

4. Finkelstein JA, Zaveri G, Wai E, Vidmar M, Kreder H, Chow E. A population-based study of surgery for spinal metastases: survival rates and complications. J Bone Joint Surg Br 2003;85:1045-50.

5. Quan GM, Vital JM, Aurouer N, et al. Surgery improves pain, function and quality of life in patients with spinal metastases: a prospective study on 118 patients. Eur Spine J 2011;20:1970-8.

6. Tomita K, Kawahara N, Kobayashi T, Yoshida A, Murakami H, Akamaru T. Surgical strategy for spinal metastases. Spine (Phila Pa 1976) 2001;26:298-306.

7. Hessler C, Burkhardt T, Raimund F, et al. Dynamics of neurological deficit after surgical decompression of symptomatic vertebral metastases. Spine (Phila $\mathrm{Pa}$ 1976) 2009;34:566-71.

8. Fisher CG, DiPaola CP, Ryken TC, et al. A novel classification system for spinal instability in neoplastic disease: an evidence-based approach and expert consensus from the Spine Oncology Study Group. Spine (Phila Pa 1976) 2010;35:E1221-9.

9. Fourney DR, Frangou EM, Ryken TC, et al. Spinal instability neoplastic score: an analysis of reliability and validity from the spine oncology study group. J Clin Oncol 2011;29:3072-7.

10. Fisher CG, Versteeg AL, Schouten R, et al. Reliability of the spinal instability neoplastic scale among radiologists: an assessment of instability secondary to spinal metastases. AJR Am J Roentgenol 2014;203:86974.

11. Campos M, Urrutia J, Zamora T, et al. The Spine Instability Neoplastic Score: an independent reliability and reproducibility analysis. Spine J 2014;14:1466-9.

12. Arana E, Kovacs FM, Royuela A, et al. Spine Instability Neoplastic Score: agreement across different 
medical and surgical specialties. Spine J 2016;16:5919.

13. Versteeg AL, Verlaan JJ, Sahgal A, et al. The Spinal Instability Neoplastic Score: impact on oncologic decision-making. Spine (Phila Pa 1976) 2016;41 Suppl 20:S231-7.

14. Zadnik PL, Hwang L, Ju DG, et al. Prolonged survival following aggressive treatment for metastatic breast cancer in the spine. Clin Exp Metastasis 2014;31:4755.

15. Zadnik PL, Goodwin CR, Karami KJ, et al. Outcomes following surgical intervention for impending and gross instability caused by multiple myeloma in the spinal column. J Neurosurg Spine 2015;22:301-9.

16. Tokuhashi Y, Matsuzaki H, Oda H, Oshima M, Ryu J. A revised scoring system for preoperative evaluation of metastatic spine tumor prognosis. Spine (Phila $\mathrm{Pa}$ 1976) 20051;30:2186-91.

17. Katagiri H, Okada R, Takagi T, et al. New prognostic factors and scoring system for patients with skeletal metastasis. Cancer Med 2014;3:1359-67.
18. Poon M, Zeng L, Zhang L, et al. Incidence of skeletalrelated events over time from solid tumour bone metastases reported in randomised trials using bone-modifying agents. Clin Oncol (R Coll Radiol) 2013;25:435-44.

19. Hussain I, Barzilai O, Reiner AS, et al. Patient-reported outcomes after surgical stabilization of spinal tumors: symptom-based validation of the Spinal Instability Neoplastic Score (SINS) and surgery. Spine J 2018;18:261-7.

20. Cavalcante RA, Fernandes YB, Marques RA, et al. Is there a correlation between the spinal instability neoplastic score and mechanical pain in patients with metastatic spinal cord compression?: a prospective cohort study. J Craniovertebr Junction Spine 2017;8:187-92.

21. Tancioni F, Navarria P, Pessina F, et al. Assessment of prognostic factors in patients with metastatic epidural spinal cord compression (MESCC) from solid tumor after surgery plus radiotherapy: a single institution experience. Eur Spine J 2012;21 Suppl 1:S146-8. 